\title{
Visual-motor efficiency (VME) and the information transmitted in visual-motor tasks
}

\author{
BARBARA SAKITT \\ Massachusetts Institute of Technology, Cambridge, Massachusetts 02139
}

\begin{abstract}
An information theory approach to the analysis of data from visual-motor experiments is suggested here. A solution to the arbitrariness of the information of continuous distributions is proposed so that the amount of information transmitted by movements of the limbs, final position, or even electromyographic activities can be calculated as absolute measures. A new index, visual-motor efficiency, is introduced, which is an absolute measure of performance. Since it is an absolute quantitative index, it can be used to compare different subjects at the same visual-motor task, to compare the same subject's performance at different tasks, to chart the progress of a subject during training, or to make virtually any absolute comparisons of efficiency.
\end{abstract}

Norbert Wiener and others, in the development of cybernetics, introduced the idea of thinking about living systems in terms of communication and control theory. The vocabulary of the engineer and physicist was adopted by the psychologist and physiologist as information theory, signal detection theory, control systems, and so on, began to be applied to human and animal data. The theme in common with all these cybernetic applications is the view of a human subject as a channel, in the information theory sense. A channel as defined by Shannon (1948) is "the medium used to transmit the signal from transmitter to receiver."

Using the ideas developed in cybernetics, a visualmotor task can be thought of as the communication of information; the visual stimulus, whether it be an array of lights in the laboratory or a panel in a flight simulator, as the information source; the person making a visually triggered movement to the stimulus, as a noisy channel; and the motor response (e.g., touching the indicated part of the panel), as the transmitted message, with all these terms being used in the exact Shannon (1948) sense.

Reviewing Shannon's (1948) idea of information transmission, and using the same notation as Shannon and Weaver (1964), but applied to a visual-motor task, let $\mathrm{H}(\mathrm{x})$ be the information in the visual display, $\mathrm{H}(\mathrm{y})$ the information in the motor responses, $\mathrm{H}(\mathrm{x}, \mathrm{y})$ the joint information, and $T(x, y)$ the information transmitted by the subject in a visual-motor task. Then

$$
T(x, y)=H(x)+H(y)-H(x, y) .
$$

For experiments in which this method is to be applied, the visual stimuli are discrete and $x_{i}$ refers to the different stimulus locations. However, the responses are essentially continuous, consisting of either final positions of movements of the arm and body or electromyographic activities of muscles. This poses a problem, since

This research was supported by Grants NS 16300 and EY 02621 from the National Institutes of Health. unlike the discrete case, the information of a continuous distribution has an arbitrary constant (Shannon, 1948) corresponding to an undefined baseline in the calculation of the information transmitted. The responses have to be divided into discrete bins, but the choice of the size of the bin can influence the amount of calculated information. This problem can be overcome so that successful quantization is possible, and the method will be described below. But for now, assume that it is possible. After so doing, the $y_{i}$ will refer to the different responses put into these discrete bins.

\section{INFORMATION TRANSMITTED-DISCRETE CASE}

For a discrete system, Shannon (1948) defined the average information per message as

$$
H=-\sum_{i} p_{i} \log _{2} p_{i},
$$

where $p_{i}$ is the probability that the ith message will occur. When there are $r$ equally probable messages, this reduces to $H=\log _{2} r$. If the information source is an array of lights, a 2-light system has 1 bit of information, a 16-light system has 4 bits, a 17-light array has 4.04 bits, and so forth.

Let $\mathrm{N}_{\mathrm{i}}$ be the number of times the ith light is presented, $N_{j}$ the number of times the $j$ th response for the arm is given, $N_{i j}$ the number of times the jth response is given to the ith light, and $\mathrm{N}$ be the total number of trials in a session. Then, substituting in the definition of information, it follows that

$$
\begin{aligned}
& H(x)=\log _{2} N-(1 / N) \sum_{i} N_{i} \log _{2} N_{i} \\
& H(y)=\log _{2} N-(1 / N) \sum_{j} N_{j} \log _{2} N_{j} \\
& H(x, y)=\log _{2} N-(1 / N) \sum_{i j} N_{i j} \log _{2} N_{i j} .
\end{aligned}
$$

Substituting these values in Equation 1 yields $\mathrm{T}(\mathrm{x}, \mathrm{y})$. 
The maximum value of $T(x, y)$, the information transmitted by the subject in the visual-motor task, is $H(x)$, the information in the stimulus. This occurs when the subject's responses are perfect, so that by merely observing the final motor responses, one could deduce which stimulus occurred. The minimum amount of information transmitted is zero and occurs when the responses are random with respect to the stimuli.

\section{VISUAL-MOTOR EFFICIENCY}

Let visual-motor efficiency (VME) be defined as the ratio of the information transmitted divided by the maximum information transfer possible. It can be converted to a percentage by multiplying by 100 .

$$
\begin{aligned}
\text { VME } & =100[T(x, y) / \text { Maximum Value of } T(x, y)] \\
& =100[T(x, y) / H(x)] .
\end{aligned}
$$

This index of visual-motor efficiency lies between 0 and $100 \%$. When the visual-motor act accurately transmits all the information about the visual stimulus, the index is $100 \%$. When it is independent of the visual stimuli, the index is 0 . Note that this efficiency index is an absolute measure of performance, because VME is the percentage relative to perfect performance. Thus, as an absolute measure, it can be used for comparisons across tasks or across subjects, during training, across stimulus conditions, across response conditions, or across virtually any conditions. Thus the index can be used to chart progress of an individual for different visual-motor tasks during training. Similarly, progress can be compared across individuals. It can be used to determine which training sequences are most effective, to determine which aspects are worth practicing and which are not, to select the optimal visual displays for training and simulation, and to preselect promising individuals. A broad range of application is possible because the VME index permits absolute quantitative comparisons between virtually all visual-motor tasks.

This novel use of information theory in visual-motor coordination is completely different from the Fitts (1954) work on movement time and motor channel capacity. It uses techniques similar to those of Attneave (1959) and others who worked in verbal judgments. What is being proposed here is that the efficiency of visual-motor performance be measured by how well a subject's movement transmits information about a visual display compared with the theoretically maximum amount.

In general, the calculation of the information transmitted requires the use of Equations 1-3. Figure 1 shows an example in which there are four stimuli and four discrete responses. For ease of exposition, it is assumed that each stimulus is presented equally often, eight times, for a total of 32 trials. The left matrix shows

\section{DISCRETE CASE}

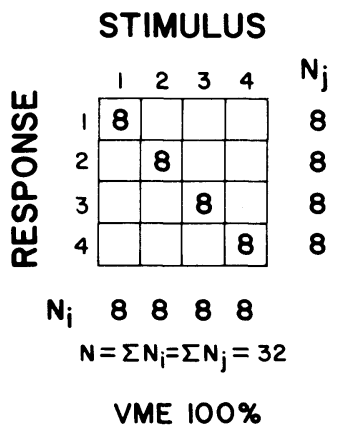

$$
\begin{aligned}
& \text { STIMULUS }
\end{aligned}
$$

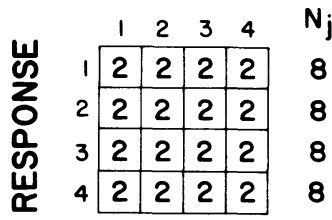

$$
\begin{aligned}
& \begin{array}{lllll}
N_{i} & 8 & 8 & 8 & 8
\end{array} \\
& N=\Sigma N_{i}=\Sigma N_{j}=32 \\
& \text { VME O\% }
\end{aligned}
$$

Figure 1. Stimulus-response matrices for the discrete case. Matrix on left is an example of perfect performance. Matrix on right is an example of random performance.

perfect performance, since the stimuli produced nonoverlapping responses. In the right matrix, the opposite extreme is shown. For each stimulus, all responses occurred equally often. It is as if the subject kept his eyes closed and randomly answered. For the left matrix, substituting in Equations 1 and 2, $\mathrm{H}(\mathrm{x})=2$ bits, and since $H(y)$ and $H(x, y)$ involve the same sums, they are each 2 bits. Therefore, $T(x, y)=2+2-2=2$ bits. Efficiency is perfect, and VME $=100 \%$.

Consider the right matrix. $H(x)$ is the same as in the previous case, since the stimuli are the same. Similarly, the response bins involve the same sum, so that $H(x)=$ $H(y)=2$. But $H(x, y)$ is different. $H(x, y)=\log _{2} 32-$ $(1 / 32)\left(2 \log _{2} 2+\ldots+2 \log _{2} 2\right)$, where there are 16 terms for $2 \log _{2} 2$, corresponding to each entry in the matrix. Therefore, $H(x, y)=4$ and $T(x, y)=0$. There is no information transmitted, and efficiency is 0 . This is intuitively expected, since the responses are independent of the stimuli.

\section{INFORMATION OF A CONTINUOUS DISTRIBUTION}

In the discrete case, the calculation of the information transmitted and the VME index are absolute measures. However, for continuous distributions, there is an intrinsic difficulty in calculating the information. For a continuous variable $\mathrm{x}$ with a density distribution function $\mathrm{p}(\mathrm{x})$, Shannon (1948) defined the information as

$$
H(x)=-\int p(x) \log _{2} p(x) d x .
$$

As pointed out by Shannon, the information in the discrete case is an absolute measure of the randomness of the variable, but in the continuous case it is relative to the coordinate system chosen, such that the entropies calculated for two different coordinate systems differ by the Jacobian of coordinate transformation.

This poses a theoretical problem for the calculation 
of the information contained in continuous responses. For example, divide the range of $\mathrm{x}$ into $\mathrm{N}$ equal intervals, each of size $\Delta x$. Let $p_{i}$ be the probability that $x$ is in the ith interval. This is equal to $\mathrm{p}\left(\mathrm{x}_{\mathrm{i}}\right) \Delta \mathrm{x}$, and the information is

$$
\begin{aligned}
H(x)= & -\sum_{i=1}^{N} p_{i} \log _{2} p_{i} \\
= & -\sum_{i=1}^{N} p\left(x_{i}\right) \Delta x \log _{2}\left[p\left(x_{i}\right) \Delta x\right] \\
= & -\sum_{i=1}^{N} p\left(x_{i}\right) \Delta x \log _{2} p\left(x_{i}\right) \\
& -\sum_{i=1}^{N} p\left(x_{i}\right) \Delta x \log _{2} \Delta x \\
= & -\sum_{i=1}^{N} p\left(x_{i}\right) \log _{2} p\left(x_{i}\right) \Delta x-\log _{2} \Delta x
\end{aligned}
$$

As $\Delta \mathrm{x}$ approaches zero, the first term approaches Equation 5 , but the second term approaches infinity. Thus the amount of information differs by $\log _{2} \Delta x$, an arbitrarily large amount. For instance, the information of a one-dimensional Gaussian distribution whose standard deviation is $\sigma$ is $\log \sqrt{2 \pi \mathrm{e}} \sigma$ (Shannon, 1948), when it is clear that the information depends upon the system of units used. This is in contrast to the discrete case, in which there is no arbitrary constant and the information is absolute. Note that Shannon's channel capacity theorem is absolutely true for the continuous case, since two information measures are subtracted from each other so that the arbitrary constant disappears. However, as seen in Equation 1, in the calculation of information transmitted, there are three information terms, so that the arbitrarily large constant causes an arbitrariness in the information transmitted. In the next section, a solution will be proposed to overcome this difficulty.

\section{SOLUTION FOR THE CALCULATION FOR THE CONTINUOUS CASE}

Consider an example, analogous to that described for the discrete case, in which the responses are continuous. Responses of interest in visually triggered movements include final limb position, integrated electromyographic activities, and so on. In such a natural case, random presentation of stimuli will result in unequal numbers of each stimulus. Specifically, consider an experiment in which a subject is required to make visually triggered movements without sight of the moving limb. The stimulus array consists of eight lights, only one of which can be lit on any trial. The presentation of the stimuli is random, so that on any trial, the probability of any particular light's being lit is $1 / 8$. Suppose that there is a total of 256 trials in one session. A typical outcome for the number of times each light is presented would be $27,34,38,31,23,31,39,33$.

To take the simplest response first, consider the final arm position when rotation about a single joint is being performed. This is the one-dimensional continuous case, since final position is an angle. Suppose that the final positions vary from 0 to $80 \mathrm{deg}$.

It is simplest to explain how discrete response bins are chosen by describing the method first and then examining the stimulus-response matrix, to show how different choices could lead to incorrect estimates of the information transmitted. The 256 different responses (joint angles, in this example) are put in order, without regard to which stimulus was presented. The smallest angles appear on the top of the list and the largest angles on the bottom. The response bins are chosen so that, if performance were perfect and monotonic, the stimulusresponse matrix would be diagonal. That is, since there were 27 presentations of Stimulus 1, the 27 smallest angles are put into Response Bin 1 . Similarly, since there were 34 presentations of Stimulus 2, the next 34 angles are put into Response Bin 2, and so on. Figure 2a shows the stimulus-response matrix that would occur for perfect performance. Since the matrix is completely diagonal, merely by observing the final arm position, one could deduce which light was lit. But a diagonal matrix always leads to $100 \%$ efficiency. Using Equation 2 , it follows that $\mathrm{H}(\mathrm{x})=-8(1 / 8) \log _{2}(1 / 8)=3$ bits of information in the stimulus. Since the matrix is diagonal, the sum for $\mathrm{H}(\mathrm{y})$ will be the same as the sum for $\mathrm{H}(\mathrm{x}, \mathrm{y})$. Hence, from Equation 1, $\mathrm{T}(\mathrm{x}, \mathrm{y})=\mathrm{H}(\mathrm{x})=$ 3 bits. Substituting in Equation 4, VME $=100 \%$ for the example of Figure 2a.

Contrast this choice with one based on equal intervals of angles, say every $10 \mathrm{deg}$. With the same data, the responses to the first stimulus might have fallen partly in the interval 0 to $10 \mathrm{deg}$ and partly in the 10 - to 20 deg interval. This choice would result in a nondiagonal matrix, implying less than perfect performance. Yet the responses to different stimuli were actually completely nonoverlapping. This illustrates how an incorrect choice
A

CONTINUOUS CASE

STIMULUS

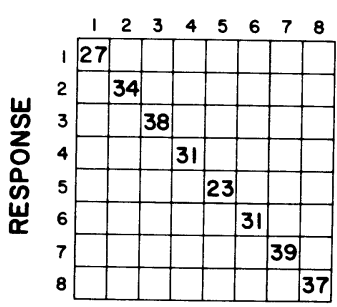

$N_{i} 2734383123313937$
$N=\sum N_{i}=\sum N_{j}=256$
B

CONTINUOUS CASE

STIMULUS
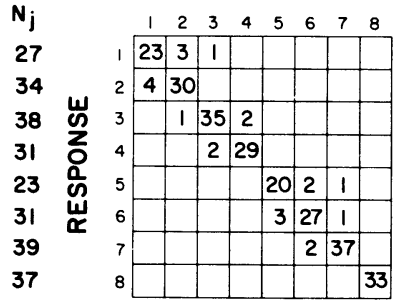

$N_{i} 2734383123313933$ $N=\Sigma N_{i}=\Sigma N_{j}=256$
Figure 2. Stimulus-response matrices for the continuous case. (a) Example of perfect performance. (b) Example of intermediate performance. See text for details. 
for the response bins can lead to an incorrect estimate of the information transmitted. Another way false estimates can occur is by choosing the response bins to be so small as to contain either zero or one item in each entry. This can lead to a serious overestimate in the information transmitted, since the nonoverlap of the responses would be due primarily to the artifact of choosing bins that are too small.

The choice of response bins presented here overcomes the difficulty of the arbitrary baseline for continuous variables in information theory. The bins are chosen not on the basis of the response intervals, but rather so that the number of response entries is equal to the number of entries in the stimulus bin. Figure $2 b$ illustrates a stimulus-response matrix that could occur for nonperfect performance. The first response bin, as discussed above, contains the first 27 angles. But since the subject was not perfect, not all of these responses were for the first stimulus. As shown in the matrix, 23 of them were for Stimulus 1, 3 were for Stimulus 2, and 1 was for Stimulus 1. Similarly, for the second response bin, not all were for the second stimulus. This nondiagonal matrix represents a more realistic example for human visual-motor performance.

Since the stimulus condition is the same as that for Figure $2 a, H(x)=3$ bits. Using the matrix in Figure $2 b$ and substituting in Equation 3, $\mathrm{H}(\mathrm{y})=3.01$ bits and
$\mathrm{H}(\mathrm{x}, \mathrm{y})=3.43$ bits. Substituting in Equation 1, $\mathrm{T}(\mathrm{x}, \mathrm{y})=$ $3.00+3.01-3.43=2.58$ bits. This corresponds to a value of $\mathrm{VME}=86 \%$. Another way of looking at this is that there are eight potential messages that could be signaled by the stimulus. The number of messages that could be transmitted by the position of the arm is 5.98 $\left(\log _{2} 5.98=2.58\right)$.

This method can be applied to any continuous response, not just the final arm position. For example, the integrated electromyographic activities can be used as the responses, as can any other variable that might be of interest. For any such response, using this method, it is possible to calculate the information transmitted and the absolute efficiency of performance.

\section{REFERENCES}

Attne ave, F. Applications of information theory to psychology. New York: Holt, 1959.

FITTS, P. M. The information capacity of the human motor system in controlling the amplitude of movement. Journal of Experimental Psychology, 1954, 47, 381-391.

Shannon, C. E. The mathematical theory of communication. Bell System Technical Journal, 1948, 27, 379-423; 623-656.

Shannon, C. E., \& Weaver, W. The mathematical theory of communication. Urbana, Ill: University of Illinois Press, 1964.

(Received for publication September 10, 1980.) 